\title{
Efficacy of shear wave elastography in predicting preeclampsia in the first trimester
}

\author{
Hicran Acar Sirinoglu ${ }^{1}$, Gulsum Uysal²* (D), Hakan Nazik² (D), \\ Basak Cingillioglu ${ }^{1}$, Simten Genc ${ }^{1}$ (D), Oya Pekin ${ }^{3}$
}

\begin{abstract}
SUMMARY
OBJECTIVE: This study aimed to investigate the predictive value of shear wave elastography (SWE) for preeclampsia (PE) in first-trimester pregnancies. METHODS: Singleton pregnant women aged 18-45 years, who underwent routine first-trimester prenatal examinations (11-13 weeks+6 days) were enrolled. Pregnancies with anterior placenta and normal first-trimester screening test results were included in the study group. The SWE measurements of six areas of the placenta were performed, and the mean value was estimated. The perinatal outcomes and the demographic data were also collected. The receiver operating characteristic curve analysis was used for the accuracy of predicting PE. RESULTS: This study consisted of 84 patients, of which 9 were diagnosed with PE during the follow-up. The mean SWE value of the PE patients was higher than that of patients with normal pregnancies $(p=0.002)$. The analysis showed that the optimal cutoff value was $7.43 \mathrm{kPa}$ to predict PE in the placentas of first-trimester pregnancies, with $88 \%$ sensitivity and $78 \%$ specificity.

CONCLUSIONS: The SWE values of the placenta in the first trimester were different between normal patients and those who are subsequently developing PE. SWE may be a suitable tool for predicting PE in pregnant women.

KEYWORDS: Shear wave elastography. Preeclampsia. High-risk pregnancy. First-trimester screening.
\end{abstract}

\section{INTRODUCTION}

Shear wave elastography (SWE) is a type of sonoelastography method that measures the stiffness and elasticity of soft tissues ${ }^{1}$. This new technique has already been used in the differential diagnosis of diseases such as hepatic fibrosis, tumors, and inflammation in musculoskeletal tissues ${ }^{1,2}$. Recently, it has become popular in screening the elasticity of the placenta in high-risk pregnancies such as those with preeclampsia (PE), intrauterine growth restriction, and placental dysfunction ${ }^{3-5}$. The application of SWE is considered safe during pregnancy, and performing this technique has already been documented in obstetrics 5,6 .
PE, a known multisystem disease, generally affects 2-5\% of pregnant women, and it is associated with a perinatal and neonatal mortality rate of $10 \% \%^{7,8}$. Many studies have been conducted to predict and prevent $\mathrm{PE}^{9-11}$. They investigated maternal factors including maternal pulse wave analysis, mean arterial blood pressure, uterine artery pulsatility index (UtA-PI), serum placental protein 13, serum pregnancy-associated plasma protein A (PAPP-A), and placental growth factor ${ }^{7-9,12}$. While the cause of PE remains an enigma, clinical and pathological studies suggest that the placenta is central in the pathogenesis of this syndrome. Instead of combining the laboratory markers, the use of a noninvasive ultrasound method for screening

\footnotetext{
'Prof. Dr. Cemil Taşçıŏlu City Hospital, Department of Obstetrics and Gynecology, Division of Perinatology - Istanbul, Turkey. ${ }^{2}$ University of Health Sciences, Adana City Training and Research Hospital, Department of Obstetrics and Gynecology - Adana, Turkey. ${ }^{3}$ Zeynep Kamil Women and Children Disease Training and Research Hospital, Department of Obstetrics and Gynecology - Istanbul, Turkey. *Corresponding author: gulsumaykut@yahoo.com Conflict of interest: the authors declare there is no conflicts of interest. Funding: none. Received on September 05, 2021. Accepted on September 06, 2021.
} 
the placenta seemed appealing. Therefore, we aimed to investigate the predictive value of SWE for PE in first-trimester single-euploid pregnancies.

\section{METHODS}

This prospective study included singleton pregnant women aged 18-45 years, who underwent routine first-trimester prenatal examination (11-13 weeks+6 days) between May 2019 and December 2019 at a tertiary hospital. Ethical documents, including the protocols as per the Declaration of Helsinki, were approved by the local Institutional Review Board (Approval number: 2019/50), and written informed consent was obtained from all patients.

Patients with normal first-trimester screening test results (single-euploid pregnancies) were enrolled in the study group. Exclusion criteria were multiple gestations, pregnancies with fetal anomalies, abnormal placental location, and uterine malformations. Patients with systemic autoimmune diseases (chronic hypertension, diabetes, hepatitis, etc.), history of PE, high blood pressure, and smoking habits were also excluded.

First-trimester prenatal examinations including nuchal translucency (NT) and UtA-PI of both maternal sides were performed as previously described ${ }^{13,14}$. Routine laboratory markers, including PAPP-A and beta-human chorionic gonadotropin ( $\beta$-hCG) for the first-trimester screening test, were also obtained. Measurements were expressed as multiples of the median (MoM) values according to the percentiles adjusted for fetal crown rump length. Additionally, SWE was performed during the ultrasonographic evaluation of the first trimester. All ultrasonographic measurements were conducted by a single perinatologist. Patients with anterior placenta were included in the study to avoid reducing the effectiveness of the method. Considering the disc-like structure of the placenta, it was divided into the following six regions for measurement: maternal right, maternal central, maternal left, fetal right, fetal central, and fetal left. The mean arithmetic value was calculated. The SWE measurements of the six areas of the placenta were performed for every patient to record the measurements from different parts of the placenta. Since the placental structure is small in the first trimester and the measurement areas were close to each other, an average value was calculated. The demographic data and the detailed medical and obstetrical history were examined and recorded during patient admission.

Perinatal outcomes, including the delivery week and birth weight, were also recorded. All patients were followed up at the perinatology clinic, and the delivery timings were organized individually based on obstetrical indications. PE was diagnosed according to the following guidelines: two measurements of diastolic blood pressure $\geq 90 \mathrm{mmHg}$ of at least $4 \mathrm{~h}$ apart in a previously normotensive woman and proteinuria $\geq 300 \mathrm{mg}$ in $24 \mathrm{~h}$; or two readings of at least " ++ " on the dipstick analysis of midstream urine or catheter specimen urine (if no 24-h urine collection is available) after 20 weeks of gestation ${ }^{15}$. The primary measurement outcome was to predict PE by using SWE in the normally detected first-trimester screening.

\section{Shear wave elastography}

SWE is a safe, noninvasive technique with the advantage of being performed in the same session as the routine antenatal scanning ${ }^{2,6}$. There is no need to wait for the results like other screening markers; SWE measurement can alleviate patient anxiety. The stiffer the tissue, the higher is the shear wave velocity (SWV). Samsung HS70A ultrasound system with a Samsung CA1-7A convex transducer was used. SWE measures alterations in tissue deformation, reflective of its elastic properties when an internal or external force is applied. Once force is applied, tissue displacement can then be displayed directly as an image property, as SWE. During the examination, patients were made to lie in the supine position, breathing levels were kept steady to prevent noise and artifacts, and a minimum pressure was applied on the transducer to eliminate misleading tissue compression. A region of interest box of $10 \times 10 \mathrm{~mm}$ was positioned on the placenta, and six measurements were obtained from the central and peripheral zones of the placenta while avoiding vascular structures (Figure 1). All measurements were obtained in $\mathrm{kPa}$ formats.

\section{Statistical analysis}

Statistical Package for the Social Sciences version 20 program (SPSS Inc., Chicago, Illinois, USA) was used for the statistical evaluation of the data obtained in this study. The continuous data were summarized as mean and standard deviation, while the categorical data were summarized as numbers and percentages. The receiver operating characteristic (ROC) curve was used to investigate the accuracy of predicting the presence of PE. According to this method, for the best test definition, the sensitivity was $100 \%$, the area under the curve (AUC) was 1 , and the diagnostic value of AUC was $\mathrm{p}<0.05$.

The sampling size was calculated based on the ability of the SWE measurements to predict PE in pregnancies by the ROC analysis. In this case, considering the structure of the ROC curve, the value of the AUC was assumed to be approximately 0.85 and the minimum sample size at $80 \%$ power at a $10 \%$ error level was determined in 76 patients. 


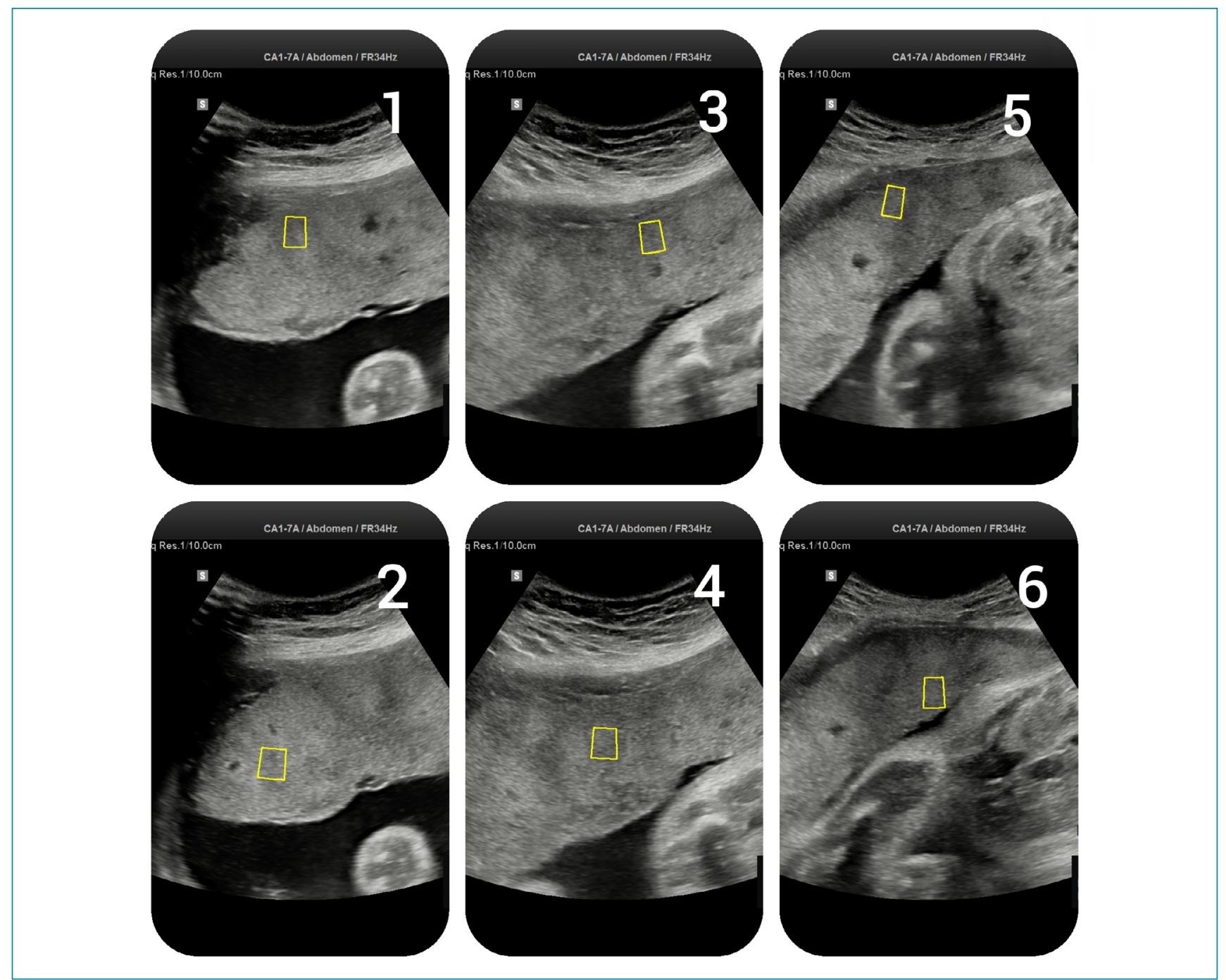

Figure 1. Placental measurements were obtained from central and peripheral zones of placenta while avoiding vascular structures. Numbers 1, 3, and 5 were maternal side and 2, 4, and 6 were fetal side; respectively.

\section{RESULTS}

This study included 113 pregnancies, of which 11 patients were lost to follow-up and 1 to abortion. All pregnancies were followed up until delivery. Patients who subsequently developed gestational diabetes mellitus, gestational hypertension, and fetal growth restriction were excluded. Since it is not clear which patient will be diagnosed with PE according to the follow-ups in the first trimester, a long time was required in terms of the follow-up period and results. The final study population consisted of 84 patients, of which 9 were diagnosed with PE later during the follow-up. The characteristics of the patients, including pregnancy outcomes and the results of screening tests, are shown in Table 1. Pregnancies with PE had earlier deliveries and lower birth weights. The mean age of the women in this study was $29 \pm 5.13$ years, and the mean gestation period was 11 weeks and 6 days. All mothers and babies were healthy, and only two babies were taken to the neonatal intensive care unit. There was no statistically significant difference between the patients with PE and those with normal pregnancies regarding age, gravidity, parity, body mass index, and the results of first-trimester screening tests, except for the mean SWE value. The mean SWE value in the PE patients was significantly higher than that of the patients with normal pregnancies $(\mathrm{p}=0.002)$. The ROC analysis for the mean values of SWE showed that the optimal cutoff value was $7.43 \mathrm{kPa}$ to predict PE in the placentas of first-trimester pregnancies, with $88 \%$ sensitivity and $78 \%$ specificity (Figure 2). Regarding the detection rates including NT, PAPP-A, $\beta$-hCG, and UtA-PI for PE, there was no better prediction parameter than the mean value of SWE. 
Table 1. Summary of characteristics of the patients, pregnancy outcomes, and the results of screening tests between groups (preeclampsia and normal pregnancies).

\begin{tabular}{|c|c|c|c|}
\hline & $\begin{array}{c}\text { Normal } \\
\text { pregnancies } \\
(n=75)\end{array}$ & $\begin{array}{l}\text { Preeclampsia } \\
\qquad(n=9)\end{array}$ & p-value \\
\hline Age (year) & $29.93 \pm 4.29$ & $26.77 \pm 7.13$ & 0.081 \\
\hline $\begin{array}{l}\text { Gravidity } \\
\text { (min-max) }\end{array}$ & $2(1-7)$ & $2(1-7)$ & 0.828 \\
\hline $\begin{array}{l}\text { Parity } \\
\text { (min-max) }\end{array}$ & $1(0-3)$ & $1(0-3)$ & 0.847 \\
\hline BMI & $25.47 \pm 2.93$ & $25.61 \pm 4.22$ & 0.926 \\
\hline Birth (weeks) & $38.66 \pm 1.09$ & $36.22 \pm 2.63$ & 0.024 \\
\hline Birth weight (g) & $3390 \pm 372$ & $2782 \pm 646$ & 0.023 \\
\hline PAPP-A (MoM) & $1.22 \pm 0.81$ & $1.05 \pm 1.01$ & 0.564 \\
\hline$\beta$-hCG (MoM) & $1.16 \pm 0.91$ & $1.20 \pm 0.99$ & 0.905 \\
\hline NT (MoM) & $0.94 \pm 0.23$ & $0.97 \pm 0.26$ & 0.736 \\
\hline \multicolumn{4}{|l|}{ UtA-PI } \\
\hline Right & $1.15 \pm 0.55$ & $1.28 \pm 0.52$ & 0.933 \\
\hline Left & $1.08 \pm 050$ & $1.28 \pm 0.61$ & 0.279 \\
\hline SWE (mean) & $6.03 \pm 2.23$ & $9.04 \pm 2.57$ & \multirow[b]{2}{*}{0.04} \\
\hline $\begin{array}{l}\text { SWE } \\
(\min -\max )\end{array}$ & $2.37-13.78$ & $3.43-11.77$ & \\
\hline
\end{tabular}

BMI: body mass index; PAPP-A: pregnancy-associated plasma protein A; MoM: multiples of the median; $\beta$-hCG: human chorionic gonadotropin; NT: nuchal translucency; UtA-PI: uterine artery pulsatility index; SWE: shear wave elastography; $p<0.05$ indicates the statistical significance.

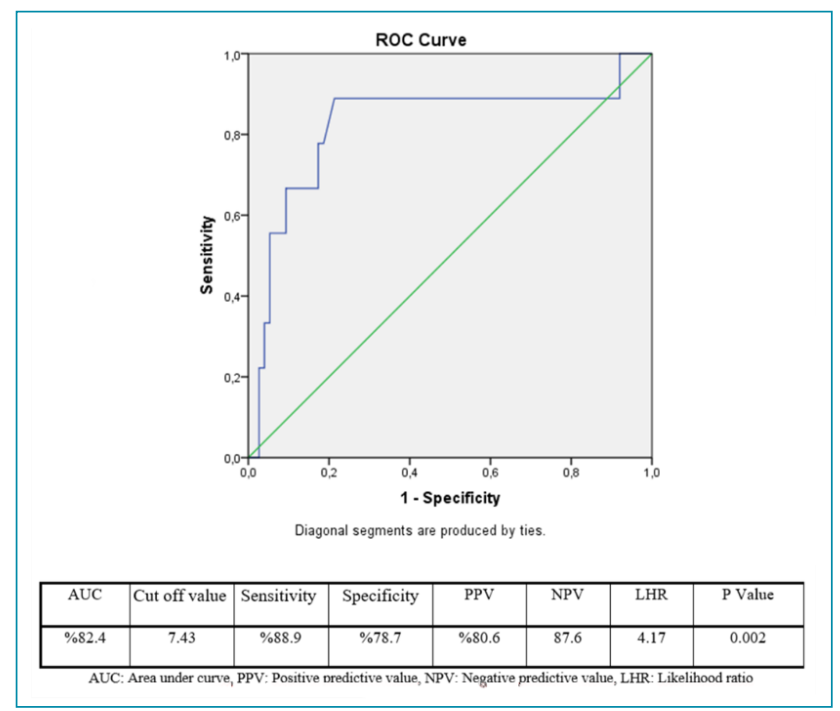

Figure 2. The receiver operating characteristic curve analysis for the positive and negative likelihood ratio of the value of $7.43 \mathrm{kPa}$.

\section{DISCUSSION}

In this study, we found that a mean SWE value above $7.43 \mathrm{kPa}$ predicted PE with very high sensitivity and specificity in low-risk pregnancy patients with normal first-trimester screening test results.

Fujita et al. ${ }^{16}$ measured point SWV to evaluate the placental elasticity for predicting the onset of PE in the second trimester. They examined 16-32 weeks of singleton pregnancies with anterior placenta and compared values between 185 low-risk and 36 high-risk patients for $\mathrm{PE}^{16}$. A higher SWV was found in the high-risk group ${ }^{18}$. Therefore, it has been suggested that SWV can be used as a parameter to predict the onset of PE. Similarly, higher placental SWV values were observed in the second- and third trimester of pregnancies with mild-severe PE or gestational hypertension as compared with the controls ${ }^{3,17}$. The UtA-PI value was found to be significantly higher in the PE group than in the controls of these studies ${ }^{3,17}$.

Cimsit et al. ${ }^{5}$ investigated SWE in the placentas of normal pregnancies $(\mathrm{n}=101)$ and those complicated with PE $(\mathrm{n}=28)$ at 20-23 weeks of gestation. A higher overall mean value of SWE was found in pregnancies complicated with PE as compared with the controls (7.01 kPa vs. $2.53 \mathrm{kPa})$. There was no statistically significant difference in the SWE values between the center and edges of the placenta in both groups. UtA notching was detected in the PE group $(60.7 \%$ bilateral and $39.2 \%$ unilateral). The SWE findings were in line with the results of our study, even in the second trimester. The efficacy of UtA-PI for the prediction of PE was found to be low and variable $^{18}$. Although the first-trimester screening tests to detect aneuploidy have become an essential part of perinatology with recent evidence of serum or ultrasound markers, their results for the prediction of PE are not impressive ${ }^{19}$.

In our study, there was no difference between the UtA-PI values and the results of the other first-trimester screening tests. Since patients with normal first-trimester screening test results were included in this study, it could explain the nonsignificant difference between the two groups. The mean SWE value in patients with PE was significantly higher than in patients with normal pregnancies $(\mathrm{p}=0.04)$. The reason could be inflammation and resistance in the fetal-maternal unit (atherosis, infarction, and hyperplasia ${ }^{20}$. PE should not be considered as a single form with different underlying pathophysiological conditions.

In a recent study ${ }^{21}$, compared with healthy pregnancies, placentas of preeclamptic pregnancies were found stiffer and more heterogeneous. Placental stiffness was not affected by the gestational age or the severity of PE. In another point of view, the process of $\mathrm{PE}$ is known to be started in the first trimester, and pharmacological intervention with medications such as low-dose aspirin is recommended to reduce the prevalence of complications. But, there is still a lack of knowledge that plays 
a major role in pathology, inflammation, or factors leading to inadequate invasion of the placenta. Both the insufficient invasion to the maternal unit and the tissue inflammation or changes were reported as stiffness in SWE in PE or fetal growth restriction. Therefore, it should be the answer to the high SWE values in our study results in the first trimester.

The major strength of our study was the SWE measurement in the first-trimester placenta and the follow-up for maternal and fetal outcomes. We believe that SWE can effectively predict PE in the first trimester.

Our study has some limitations. First, the posterior placenta was not evaluated due to the technical limitations of the SWE. Second, the distribution of PE cases was appeared to be higher than that of normal cases because the study was conducted in a tertiary hospital. Moreover, the study may be improved with larger patient numbers.

\section{CONCLUSIONS}

The SWE values differed between normal pregnancies and those that are subsequently developing PE in the first trimester. SWE may be a suitable tool, and we suggest its use clinically to predict PE.

\section{AUTHORS" CONTRIBUTIONS}

HAS: Conceptualization, Funding acquisition, Methodology, Resources, Software, Supervision, Writing - original draft. GU: Conceptualization, Project administration, Resources, Writing - original draft, Writing - review \& editing. HN: Data curation, Investigation, Visualization, Writing - review \& editing. BC: Data curation, Investigation, Validation. SG: Formal analysis, Project administration, Validation. OP: Formal analysis, Funding acquisition, Visualization.

\section{REFERENCES}

1. Edwards C, Cavanagh E, Kumar S, Clifton V, Fontanarosa D. The use of elastography in placental research - a literature review. Placenta. 2020;99:78-88. https://doi.org/10.1016/j. placenta.2020.07.014

2. Bamber J, Cosgrove D, Dietrich CF, Fromageau J, Bojunga J, Calliada $F$, et al. EFSUMB guidelines and recommendations on the clinical use of ultrasound elastography. Part 1: basic principles and technology. Ultraschall Med. 2013;34(2):16984. https://doi.org/10.1055/s-0033-1335205

3. Karaman E, Arslan H, Çetin O, Sahin HG, Bora A, Yavuz $A$, et al. Comparison of placental elasticity in normal and pre-eclamptic pregnant women by acoustic radiation force impulse elastosonography. J Obstet Gynaecol Res. 2016;42(11):1464-70. https://doi.org/10.1111/jog.13078

4. Habibi HA, Davutoglu EA, Kandemirli SG, Aslan M, Ozel A, Ucar AK, et al. In vivo assessment of placental elasticity in intrauterine growth restriction by shear-wave elastography. Eur J Radiol. 2017;97:16-20. https://doi.org/10.1016/j. ejrad.2017.10.007

5. Cimsit C, Yoldemir T, Akpinar IN. Shear wave elastography in placental dysfunction: comparison of elasticity values in normal and preeclamptic pregnancies in the second trimester. J Ultrasound Med. 2015;34(1):151-9. https://doi.org/10.7863/ ultra.34.1.151

6. Akbas M, Koyuncu FM, Artunç-Ülkümen B, Akbas G. The relation between second-trimester placental elasticity and poor obstetric outcomes in low-risk pregnancies. J Perinat Med. 2020;49(4):468-73. https://doi.org/10.1515/ jpm-2020-0464

7. Poon LC, Shennan A, Hyett JA, Kapur A, Hadar E, Divakar H, et al. The International Federation of Gynecology and Obstetrics (FIGO) initiative on pre-eclampsia: a pragmatic guide for firsttrimester screening and prevention. Int J Gynaecol Obstet. 2019;145 Suppl 1(Suppl 1):1-33. https://doi.org/10.1002/ ijgo.12802

8. Lisonkova S, Sabr Y, Mayer C, Young C, Skoll A, Joseph KS. Maternal morbidity associated with early-onset and late-onset preeclampsia. Obstet Gynecol. 2014;124(4):771-81. https:// doi.org/10.1097/AOG.0000000000000472

9. O'Gorman N, Wright D, Poon LC, Rolnik DL, Syngelaki A, Wright $A$, et al. Accuracy of competing-risks model in screening for pre-eclampsia by maternal factors and biomarkers at 11-13 weeks' gestation. Ultrasound Obstet Gynecol. 2017;49(6):751-5. https://doi.org/10.1002/uog.17399

10. Park FJ, Leung CHY, Poon LCY, Williams PF, Rothwell SJ, Hyett JA. Clinical evaluation of a first trimester algorithm predicting the risk of hypertensive disease of pregnancy. Aust $\mathrm{N} \mathrm{Z} \mathrm{J}$ Obstet Gynaecol. 2013;53(6):532-9. https://doi.org/10.1111/ ajo. 12126

11. Khalil A, Cowans NJ, Spencer K, Goichman S, Meiri H, Harrington K. First-trimester markers for the prediction of pre-eclampsia in women with a-priori high risk. Ultrasound Obstet Gynecol. 2010;35(6):671-9. https://doi.org/10.1002/ uog.7559

12. Chau K, Hennessy A, Makris A. Placental growth factor and pre-eclampsia. J Hum Hypertens. 2017;31(12):782-6. https:// doi.org/10.1038/jhh.2017.61

13. Martin AM, Bindra R, Curcio P, Cicero S, Nicolaides KH. Screening for pre-eclampsia and fetal growth restriction by uterine artery Doppler at 11-14 weeks of gestation. Ultrasound Obstet Gynecol. 2001;18(6):583-6. https://doi. org/10.1046/j.0960-7692.2001.00594.x

14. Spencer K, Souter V, Tul N, Snijders R, Nicolaides KH. A screening program for trisomy 21 at 10-14 weeks using fetal nuchal translucency, maternal serum free beta-human chorionic gonadotropin and pregnancy-associated plasma protein-A. Ultrasound Obstet Gynecol. 1999;13(4):231-7. https://doi.org/10.1046/j.1469-0705.1999.13040231.x

15. Brown MA, Lindheimer MD, Swiet M, Van Assche A, Moutquin $J M$. The classification and diagnosis of the hypertensive disorders of pregnancy: statement from the International Society for the Study of Hypertension in Pregnancy (ISSHP). Hypertens Pregnancy. 2001;20(1):IX-XIV. https://doi.org/10.1081/ PRG-100104165 
16. Fujita Y, Nakanishi TO, Sugitani M, Kato K. Placental elasticity as a new non-invasive predictive marker of pre-eclampsia. Ultrasound Med Biol. 2019;45(1):93-7. https://doi.org/10.1016/j. ultrasmedbio.2018.09.007

17. Alan B, Tunç S, Agacayak E, Bilici A. Diagnosis of pre-eclampsia and assessment of severity through examination of the placenta with acoustic radiation force impulse elastography. Int J Gynaecol Obstet. 2016;135(1):43-6. https://doi. org/10.1016/j.ijgo.2016.03.037

18. Pedrosa AC, Matias A. Screening for pre-eclampsia: a systematic review of tests combining uterine artery Doppler with other markers. J Perinat Med. 2011;39(6):619-35. https://doi. org/10.1515/jpm.2011.077
19. Halscott TL, Ramsey PS, Reddy UM. First trimester screening cannot predict adverse outcomes yet. Prenat Diagn. 2014;34(7):668-76. https://doi.org/10.1002/pd.4407

20. Nelson DB, Ziadie MS, McIntire DD, Rogers BB, Leveno KJ. Placental pathology suggesting that preeclampsia is more than one disease. Am J Obstet Gynecol. 2014;210(1):66. e1-7. https://doi.org/10.1016/j.ajog.2013.09.010

21. O'Gorman N, Wright D, Poon LC, Rolnik DL, Syngelaki A, Wright $A$, et al. Accuracy of competing-risks model in screening for pre-eclampsia by maternal factors and biomarkers at 11-13 weeks' gestation. Ultrasound Obstet Gynecol. 2017;49(6):751-5. https://doi.org/10.1002/ uog. 17399 